\title{
Recuperación del pastizal natural en un bosque nativo degradado del Espinal argentino mediante el control químico aéreo de arbustivas
}

\section{Recovery of the natural grassland in a degraded native forest of the Argentine Espinal through aerial chemical control of shrubs}

\section{Julián Alberto Sabattini ${ }^{1 *}$, Rafael Alberto Sabattini ${ }^{1}$, Florencia Urteaga Omar ${ }^{2}$, Mauricio Bacigalupo ${ }^{3}$, Juan Carlos Cian ${ }^{4}$, Iván Alberto Sabattini ${ }^{4}$ y Víctor Manuel Dopazo ${ }^{5}$}

\author{
${ }^{1}$ Universidad Nacional de Entre Ríos, Facultad de Ciencias Agropecuarias, Ecología de Sistemas Agropecuarios. Ruta 11 \\ km 10,5 E3100XAD Oro Verde, Entre Ríos, República Argentina. \\ ${ }^{2}$ Universidad Nacional de Entre Ríos, Facultad de Ciencias Agropecuarias, Estadística y Diseño Experimental. Entre Ríos, \\ República Argentina. \\ ${ }^{3}$ Dow Agroscience, Development for Range \& Pastures Argentina. Entre Ríos, República Argentina. \\ ${ }^{4}$ Universidad Nacional de Entre Ríos, Facultad de Ciencias Agropecuarias. Entre Ríos, República Argentina. \\ ${ }^{5}$ Las Taperitas S.A. Entre Rios, Argentina.
}

\author{
*Autor para correspondencia: \\ juliansabattini@fca.uner.edu.ar, \\ juliansabattini@hotmail.com \\ Conflicto de interés: \\ Los autores declaran no tener \\ conflicto de interés. \\ Licencia: \\ Artículo publicado en acceso \\ abierto con una licencia Creative \\ Commons CC-BY \\ Historial: \\ Recibido: $17 / 05 / 18$; \\ Aceptado: 01/07/19 \\ Periodo de Publicación \\ Julio - Diciembre de 2019
}

\begin{abstract}
RESUMEN
La estabilidad de los ecosistemas está directamente relacionada con un alto valor de biodiversidad $y$, en este sentido, los efectos antrópicos han generado condiciones negativas sobre los pastizales naturales. El objetivo del trabajo fue evaluar la respuesta del pastizal natural de un bosque nativo degradado del Espinal argentino con arbustivas por medio de un control químico aéreo en un sistema ganadero con pastoreo rotativo de baja carga instantánea. El estudio se realizó en el Área Natural Protegida Reserva de Usos Múltiples Estancia "El Carayá" ubicada en el centro-norte de Entre Ríos, Argentina. Se realizaron 16 evaluaciones desde el año 2012 al 2015 sobre el sector tratado químicamente con Picloram+2,4-D y tres sectores sin tratamiento. En cada evaluación se cuantificó la cobertura vegetal, la composición florística de las especies forrajeras más representativas y la biomasa vegetal, comparadas mediante pruebas no paramétricas. Los resultados de este trabajo comprueban que la utilización de una técnica de control químico aéreo aumentó significativamente, un 44\%, la cobertura y biomasa en las áreas tratadas de las especies forrajeras respecto al testigo. El control químico sobre las arbustivas persistió en el tiempo con una efectividad considerable durante los años de evaluación. Por otro lado, la composición florística de las especies herbáceas no presentó diferencias significativas entre tratamientos.
\end{abstract}

Palabras clave: Baccharis spp., malezas, control químico aéreo, arbustivas.

\begin{abstract}
The stability of the ecosystems is directly related to a high value of biodiversity and, in this sense, the anthropic effects have generated negative conditions on the natural pastures. The aim of this work was to evaluate the recovery of the natural grassland of a degraded native forest of the Argentine Spinal with shrubs by means of an aerial chemical control in a cattle system with rotary grazing of low instantaneous load. The study was carried out in the Protected Natural Area Reserve of Multiple Uses Estancia "EI Carayá" located in the north central of Entre Ríos, Argentina.16 evaluations were carried out (between 2012 and 2015) on the chemically treated sector with Picloram+2,4-D and on three sectors without treatment. In each evaluation, the vegetation cover, the floristic composition of the most representative forage species, and the plant biomass, were quantified. The results of this work show that the use of an aerial chemical control technique increases significantly, by $44 \%$, the coverage and biomass of the forage species in the treated areas with respect to the control. Chemical control over shrubs persisted over time with considerable effectiveness during the years of evaluation. Likewise, the floristic composition of the herbaceous species did not present significant differences between treatments.
\end{abstract}

Key words: Baccharis spp., weeds, aerial chemical control, shrubs. 


\section{INTRODUCCIÓN}

Los pastizales, matorrales, bosques y sabanas son biomas utilizados por el pastoreo vacuno dado que ocupan el $50 \%$ de la superficie terrestre (Bailey \& Ropes, 1998; Steinfeld et al. (2006). En particular, los bosques nativos representan el 30\% (FAO, 2007) $y$, se ha cuantificado su acelerada disminución (Hansen et al., 2013). En este sentido, América del Sur sufrió la mayor pérdida de bosques nativos, estimada en cuatro millones de hectáreas durante el período 2000 a 2005 (FAO, 2007). La Argentina no escapa a este fenómeno, estimando una pérdida de las dos terceras partes del patrimonio forestal debido al avance de la agricultura (Cozzo, 1979; FAO, 2009).

Estos biomas, proporcionan de 30 a $35 \%$ de la productividad primaria neta terrestre sustentando gran parte de la producción ganadera mundial (Safriel \& Adeel, 2005; Reynolds, Maestre, Stafford \& Lambin, 2007) brindando, además, una variedad de servicios ecosistémicos como el secuestro de carbono, mantenimiento y conservación de la diversidad genética, entre otros (Anadón, Sala, Turner \& Bennett, 2014). El aumento de la cobertura de especies leñosas nativas y exóticas sobre estos sistemas naturales conlleva un reemplazo de las especies herbáceas y una disminución de la accesibilidad y de la oferta forrajera para el ganado bovino (Archer, 1990; Moleele, Ringrose, Matheson \& Vanderpost, 2002; Graz, 2008; Tighe, Reid, Wilson \& Briggs, 2009).

Es sabido que la estabilidad de los ecosistemas está directamente relacionada con un alto valor de biodiversidad (Tilman, Hill \& Lehman, 2006) y, en este sentido, los efectos antrópicos han generado condiciones negativas sobre los pastizales naturales del mundo (Helm et al., 2009). La ocurrencia de un disturbio en una población o comunidad vegetal comprende la eliminación parcial o total de la biomasa (Grime, 1977) y se relaciona con la actividad de los herbívoros, ente otros aspectos (Villalobos, 2013). En este sentido, el sobrepastoreo de herbívoros exóticos puede modificar la composición de las comunidades vegetales nativas aumentando la proporción de plantas exóticas herbáceas y arbustivas (Hobbs \& Huenneke, 1992; Holmgren, 2002; Zimdahl, 2004; Parker, M.A., Malek \& Parker, I.M., 2006). Cuando las especies exóticas aumentan su cobertura relativa afectan a la productividad primaria del pastizal (Vitousek \& Walker, 1989; Kearns, Inouye \& Waser, 1998;
Wilcox \& Thurow, 2006). Algunos autores indican como causa del deterioro a la introducción de herbívoros, por lo que su remoción o disminución de la intensidad de pastoreo sería la técnica suficiente para la recuperación de plantas nativas (Pettit, Froend \& Ladd, 1995; Donlan, Tershy \& Croll, 2002; Beever, Wilkening, McIvor, Weber \& Brussard, 2008). Sin embargo, otros estudios muestran que la exclusión del pastoreo no mejora ni aumenta la riqueza de especies nativas ni disminuye la cobertura de especies invasoras exóticas (Stohlgren, Schell \& Vanden Heuvel, 1999).

A nivel mundial, las arbustivas han sido motivo de preocupación por mucho tiempo por parte de los productores ganaderos debido al previsible impacto negativo sobre la producción secundaria (Scholes y Archer, 1997), por lo que se han implementado técnicas para su control. En la actualidad más del $70 \%$ del territorio argentino está presenta alta cobertura de arbustivas; caracterizadas por tener un gran poder de adaptación al medio ambiente $y$ un efecto selectivo sobre los animales en pastoreo (Anadón et. al., 2014). Las arbustivas compiten con las especies del estrato herbáceo por luz, agua y nutrientes provocando una rápida invasión dentro de un potrero dificultando el manejo del rodeo (Böker, Gulielmetti y Knudtsen, 1989).

A principios de siglo $X X$ la superficie ocupada por bosques nativos en la provincia de Entre Ríos era de 2,5 millones de hectáreas, y estimaciones recientes indican que la superficie boscosa no supera 1,5 millones de hectáreas (Sabattini, J.A., Sabattini, R.A. y Ledesma, 2015). En estos ecosistemas se desarrolla gran parte de la ganadería de cría bovina de la provincia, pero los mismos se encuentran altamente degradados debido al sobrepastoreo bovino, provocando la arbustización, la erosión en cárcavas, y la presencia de suelo desnudo (Casermeiro, Spahn, De Petre, 2001; Sabattini, Muzzachiodi y Dorsch, 2002).

En particular, el proceso de arbustización de los bosques del Espinal Argentino se verifica mediante la invasión del complejo de chilcas (Baccharis spp. L. y Eupatorium spp. L.), Aloysia gratissima (Gillies \& Hook) Tronc., Eryngium horridum Malme, Melica macra Nees, entre otras especies (Sione, Sabattini, Ledesma, Dorsch y Fortini, 2006). Sabattini et al (2015) han registrado que los departamentos del centro-norte de la provincia de Entre Ríos presentan una cobertura de arbustivas superior al 50\%, destacándose Baccharis punctulata L., $A$. 
gratissima, B. coridifolia DC., E. bunifolium R.M.King \& H.Rob., Trithrinax campestris Drude \& Griseb., B. notosergilla L., Senecio grisebachii Baker, y Opuntia ficus indicus (L.) Mill.

Una ventaja ecológica de estas malezas es su gran capacidad de rebrotar y semillar invadiendo nuevamente el territorio. En este sentido, experiencias realizadas en estos ambientes han demostrado la efectividad de tales prácticas, traducido en una mayor cobertura de las especies vegetales palatables $y$, por ende, una tendencia favorable de la calidad del pastizal natural y en un incremento del $15-20 \%$ en la superficie de pastoreo (Sabattini et al., 2002). Las herramientas para el control de estas especies invasoras son variadas, desde técnicas mecánicas, biológicas, físicas y hasta el control químico.

Los métodos químicos se basan en la aplicación de herbicidas selectivos de acción sistémica, como Picloram, Tryclopir o Clopyralid, presentando alta susceptibilidad cuando se realiza una aplicación adecuada que consiste en seleccionar la época del año, determinar la susceptibilidad de las especies y determinar el modo de aplicación (Martín, 2014). En su mayoría estos activos presentan un modo de acción de tipo hormonal, es decir deben ser absorbidos y transportados a los órganos vegetales para que produzcan la remisión y posterior muerte de la planta. Por tal motivo requieren ser aplicados en la época correcta, siendo óptima cuando hay mayor flujo de fotosintatos producidos por la planta (Fumagalli y Kunst, 2002).

Estudios en otras regiones del mundo indican el modo de aplicación, el principio activo y la eficiencia de control sobre especies arbustivas (Holmes, 1998; Sabattini, R.A., Ledesma, Sione, Fontana y Sabattini, J.A., 2011; Gann, Thompson \& Schuler, 2012; Renz, 2013; Enloe \& Loewenstein, 2013; Tolozano, Pisani y Puricelli, 2017) leñosas (Randall, 2013), y herbáceas acuáticas (Getsinger, Madsen, Koschnick, \& Netherland, 2002; Martín, 2014) que son invasoras en ecosistemas naturales. Teniendo en cuenta lo mencionado, el objetivo del trabajo fue evaluar la respuesta del pastizal natural de un bosque nativo degradado del Espinal argentino con arbustivas por medio de un control químico aéreo en un sistema ganadero con pastoreo rotativo de baja carga instantánea.

\section{MATERIALES Y MÉTODOS}

El estudio se realizó en el Área Natural Protegida (ANP) - Reserva de Usos Múltiples Estancia "El Carayá" (ANP Carayá) ubicada en el centro-norte de Entre Ríos, Argentina (Figura 1). Según Rojas y Saluso (1987), la región corresponde al clima templado húmedo de llanura, siendo la temperatura media anual de $18,9^{\circ} \mathrm{C}$ (INTA, 1986). La precipitación media anual es de $1.300 \mathrm{~mm}$, concentradas entre los meses de Octubre y Marzo (1967-2014).
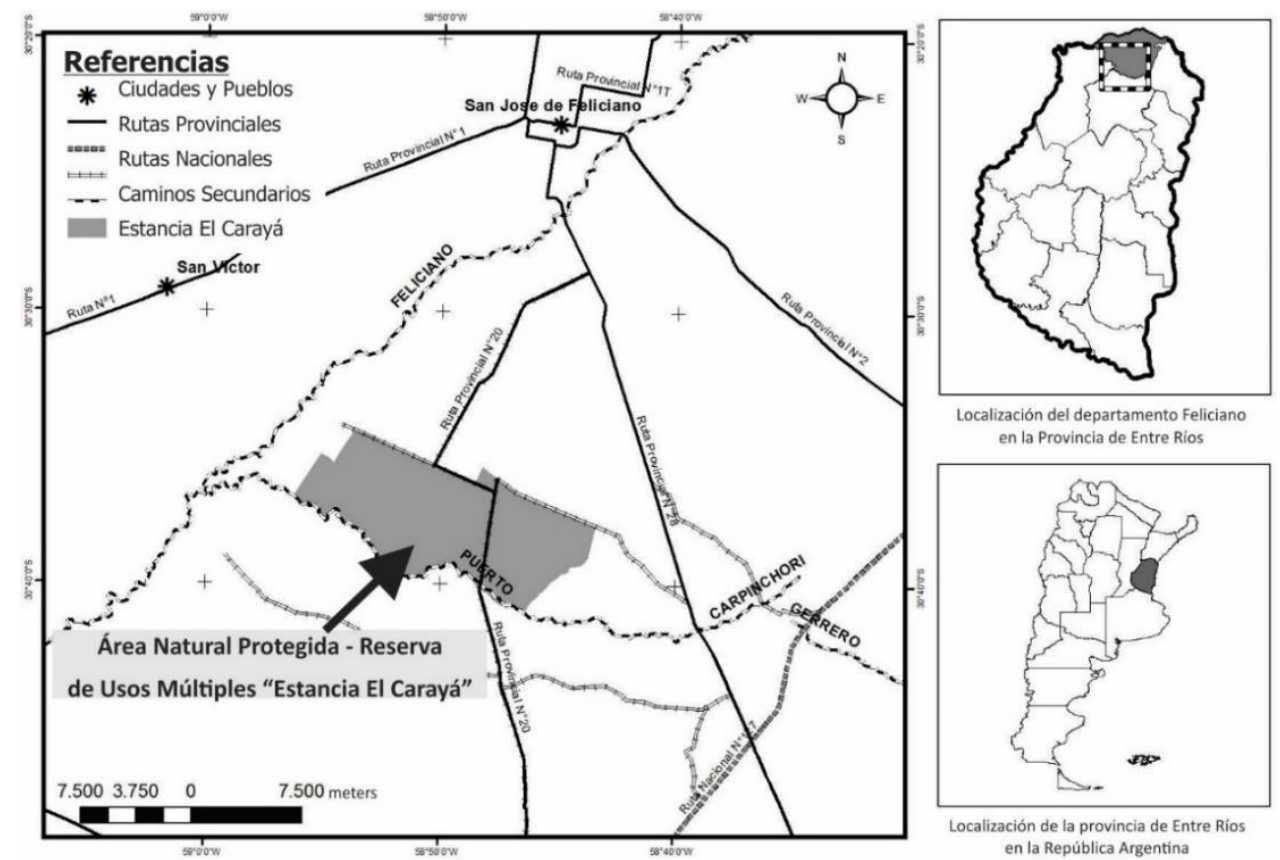

Figura 1. Localización del área de estudio en la provincia de Entre Ríos (República Argentina). 
Durante el período de evaluación se registraron cambios en la distribución de las precipitaciones mensuales y anuales.

En el año 2012 y 2014 se observó un aumento del $38 \%$ en comparación con las precipitaciones medias anuales de los últimos 30 años (1982-2011) en el ANP-El Carayá (Figura 2). Sin embargo, durante el período otoño-invierno del 2013, las precipitaciones fueron inferiores a las normales. Los suelos están ubicados topográficamente en zonas planas a muy suavemente onduladas con pendientes inferiores al
$1 \%$, son imperfectamente drenados y después de las Iluvias quedan con exceso de agua en superficie (Sabattini et al., 2015). Tienen un epipedón de poco espesor de textura limosa al que le sigue un horizonte impermeable y con dificultad para la penetración de las raíces, de textura franca arcillo limosa.

No presentan peligro de erosión hídrica, pero el exceso periódico de agua en superficie y las características físico-químicas adversas, reducen su aptitud y capacidad productiva (INTA, 1980).

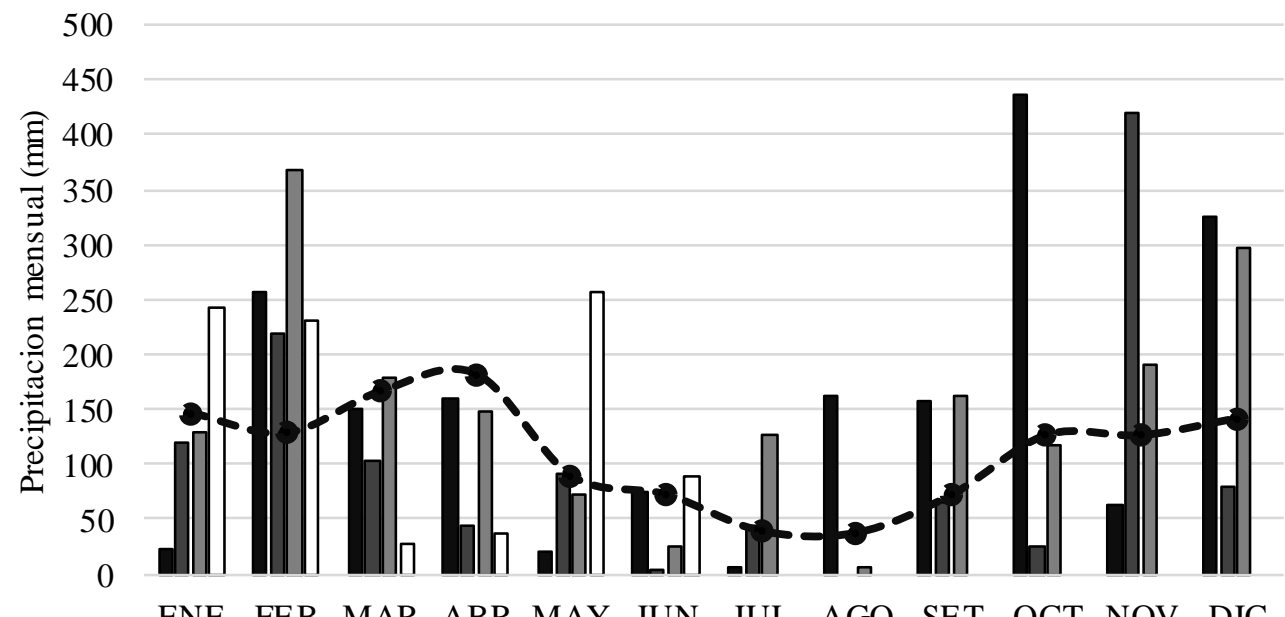

ENE FEB MAR ABR MAY JUN JUL AGO SET OCT NOV DIC

$2012 \longleftarrow 2013 \longleftarrow 2014 \longleftarrow 2015$ - - Media Histórica 1982-2011

Figura 2. Distribución de las precipitaciones mensuales históricas (1982-2011) y de los años 2012 a 2015 del Área Natural Protegida Reserva de Usos Múltiples Estancia "El Carayá".

El área de estudio presenta como suelo dominante a la Asociación Serie Arroyo Quebracho y Serie Garat correspondientes a la Serie Arroyo Quebracho (Ocracualfe vértico) y la Serie Garat (Argiuacuoles vérticos), con dominancia de las características de Arroyo Quebracho. En esta unidad cartográfica se evaluaron contenidos de fósforo ligeramente más altos respecto al resto de la región, como también muy provistos de materia orgánica coincidente con alta cobertura de la vegetación, fundamentalmente de arbustivas (Sabattini et al., 2015).

El área de estudio corresponde a la provincia Fitogeográfica del Espinal (Oyarzabal et al., 2018), que se caracteriza por bosques xerófilos dominados por Prosopis nigra Griseb., P. affinis Spreng. y Vachellia caven Molina. Los bosques nativos son heterogéneos por la fisonomía y estructura del estrato arbóreo, y por el desarrollo del estrato arbustivo, a causa de la diversidad en los suelos y la profusa hidrográfica que surca la región. En general, el estado actual de estos ecosistemas se caracteriza por la ubicación dentro de la sucesión vegetal, siendo los bosques secundarios dominantes en extensión en el área de estudio con un 20 a $25 \%$ de cobertura vegetal, que corresponden a etapas intermedias en las que mejora la diversidad, pero aún no se estabiliza (Sabattini, Wilson, Muzzachiodi y Dorsch, 1999). Estudios realizados en ANP-Carayá indican la presencia de Celtis ehrenbergiana Gillies ex Planch., Geofroea decorticans (Gill. ex Hook. \& Arn.) Burkart, Eugenia cisplatensis Cambess. y Parkinsonia aculeata L., como especies acompañantes del estrato arbóreo, como también de numerosos individuos juveniles de $V$. caven y $P$. affinis secos y muertos en pie. La denominación "cerrados" responde a la cobertura de arbustivas entre el $32,5 \%$ y $62,5 \%$, siendo la especie 
dominante el Baccharis punctulata L., acompañada de Eupatorium buniifolium Hook. \& Arn., E. laevigatum Lam. y Aloysa grattisima (Gillies \& Hook. ex Hook.) Tronc. La condición del pastizal es excelente (Sabattini et al., 2002) con dominancia de especies de los géneros Nassella E. Desv., Bothriochloa Kuntze, Chloris Sw. y Paspalum L., siendo muy palatables, de buen porte y vigor ante el pastoreo de ganado bovino. El sitio estudiado presentó abundante material vegetal seco sobre el suelo como variables de alteración dominante, como evidencias de fuego sobre los árboles (Sabattini et al., 2015).

La aplicación química se realizó el 20/04/2012 en el Lote 4 Sureste A (30०37'24,20"LS $\left.58^{\circ} 48^{\prime} 36,98^{\prime \prime L O}\right)$ sobre una superficie de 85 ha. El herbicida seleccionado fue Picloram 11,4 g + 2,4-D $44,7 \mathrm{~g}$, utilizando $4 \mathrm{~L} \mathrm{ha}^{-1}$ de producto comercial y $1 \mathrm{~L} \mathrm{ha}^{-1}$ de coadyuvante metilado. Ambos compuestos fueron disueltos en agua, aplicando un caldo de $40 \mathrm{~L} \mathrm{ha}^{-1}$. El herbicida es sistémico para el control de malezas de hoja ancha y renovales, presentando una selectividad que excluye su control a gramíneas y árboles superiores con $10 \mathrm{~cm}$ de diámetro a la altura del pecho.

El sistema de aplicación fue aéreo con avión, pulverizando con gotas entre 200 a $250 \mu$ de diámetro para garantizar la adherencia, penetración sobre las hojas y disminuir el riesgo de deriva. La aplicación comenzó a las 7:45 h con una leve brisa (menor a $5 \mathrm{~km} / \mathrm{h}$ ) y alto contenido de humedad (mayor al 70\% HR), finalizando a las 9:30 h. En el sector Este del Lote se ubicó la situación Testigo (sin aplicación) sobre 4 ha.

Se localizaron aleatoriamente seis sitios de evaluación con parcelas rectangulares de $250 \mathrm{~m}^{2}$ (25 m de largo y $10 \mathrm{~m}$ de ancho): tres sobre el sector tratado químicamente (Q) y tres sectores sin tratamiento (T). Para evaluar la dinámica de la recuperación del pastizal natural se realizaron 16 evaluaciones desde el año 2012 al 2015: 16/05, 06/07, 13/09, 15/11/2012; 16/03, 24/05, 26/07, $21 / 09$ у $17 / 12 / 2013 ; 01 / 03,19 / 05,19 / 07$ у $13 / 10 / 2014 ; 04 / 01,13 / 03$ y 03/06/2015.

En cada evaluación se cuantificó la cobertura vegetal, la composición florística de las especies forrajeras más representativas y la biomasa vegetal. La cobertura vegetal se determinó con el método de intercepción de la línea de Canfield (Matteucci y Colma, 1982), considerando las siguientes fracciones: forrajeras (especies herbáceas monocotiledóneas, dicotiledóneas y ciperáceas palatables); B. punctulata, E. horridum, y otras especies herbáceas no forrajeras (ONF). Para las mediciones se utilizaron tres transectas fijas de 30 metros dentro de cada parcela en cada tratamiento (tres en sector tratado químicamente y tres en el testigo). Cada transecta se dividió en 10 partes y se evaluó la cobertura de cada fracción.

Para cuantificar la composición florística de las especies herbáceas forrajeras se utilizó el método de Braun-Blanquet (1979), evaluando la abundancia y cobertura de la vegetación por medio de la siguiente escala: $r$ (individuos solitarios con baja cobertura), + (pocos individuos con baja cobertura), 1 (menor al 5\% de cobertura), 2 (entre el 5 y $25 \%$ ), 3 (entre 25 y $50 \%$ ), 4 (entre 50 y $75 \%$ ) y 5 (mayor al $75 \%$ de cobertura). Se realizaron tres repeticiones por cada tratamiento en cada evaluación, tomando $1 \mathrm{~m}^{2}$ como tamaño muestral.

La biomasa en cada tratamiento se midió por medio del corte vegetal en las siguientes fracciones: forrajeras, B. punctulata, E. horridum, y ONF. La altura de corte fue de $5 \mathrm{~cm}$ del suelo tomando un área de corte de $1 \mathrm{~m}^{2}$. El material verde se pesó con una balanza portátil de un decimal de precisión y luego se realizó el secado en estufa a $80^{\circ} \mathrm{C}$ durante $48 \mathrm{~h}$. Posteriormente se pesó el material seco para cuantificar la producción de biomasa vegetal en términos de $\mathrm{kg} \mathrm{MS} \mathrm{ha-1.} \mathrm{Durante} \mathrm{el} \mathrm{ensayo} \mathrm{se} \mathrm{realizó}$ un pastoreo rotativo de bovinos de cría en función de la oferta de forraje de acuerdo con la época del año con una carga animal entre 0,4 a 0,7 EV/ha/año.

Las diferencias en la cobertura y biomasa entre los sectores tratados químicamente (Q) y testigo $(T)$ se calcularon mediante la Prueba U de Mann-Whitney, dado que no cumplían con los supuestos de normalidad y homogeneidad de varianzas. Para el análisis de la composición florística se utilizó la prueba no paramétrica de Kruskall-Wallis (K-W). En todos los casos, se analizaron las diferencias entre tratamiento considerando el periodo evaluado, y además para cada año por separado.

\section{RESULTADOS Y DISCUSIÓN}

\section{Cobertura vegetal}

Al finalizar el período de evaluación se observó un aumento del $44 \%$ en la cobertura de las especies 
herbáceas forrajeras en el sector tratado respecto al testigo $(Z=-6,060 ; p<0,05)$. Este aumento significativo en la cobertura de especies forrajeras se atribuye a disminuciones importantes de las fracciones $B$. punctulata y ONF luego de la aplicación química en las áreas tratadas, obteniendo un porcentaje de control promedio de $82 \%(Z=-6,453$, $\mathrm{p}<0,05)$ y $44 \% \quad(Z=-5,180, \quad p<0,05)$, respectivamente. Sin embargo, la cobertura de $E$. horridum no presentó diferencias significativas entre tratamientos $(Z=-1,388 ; p=0,165)$ luego de 1.139 días después de aplicación 'DDA' (Figura 3).

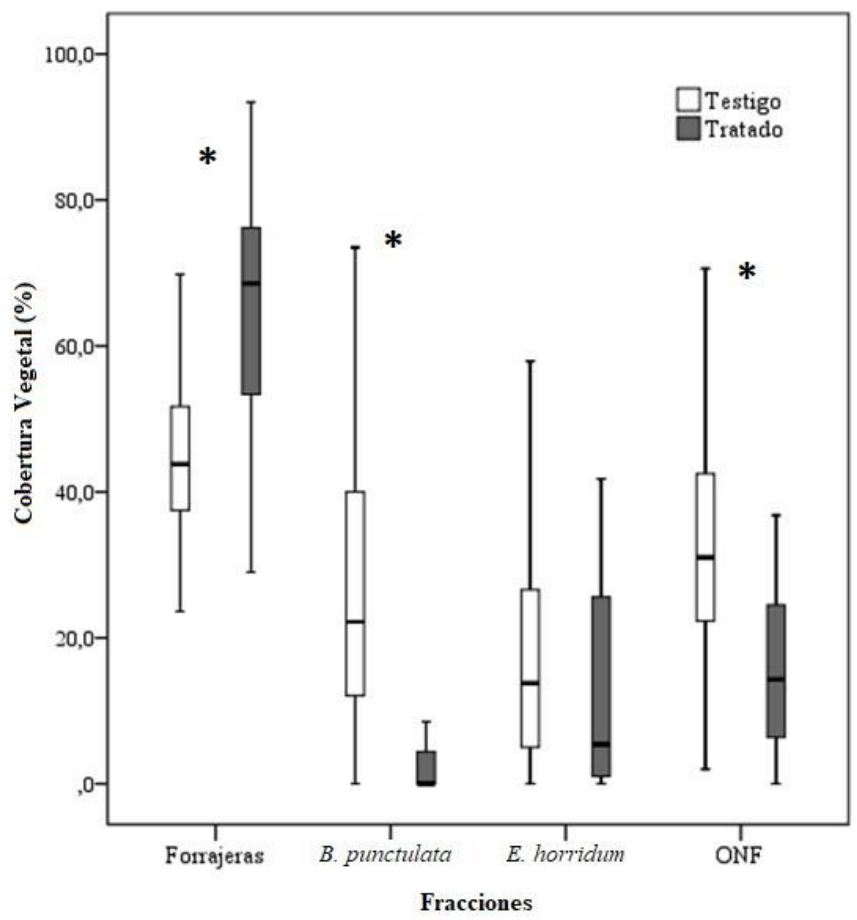

Figura 3. Cobertura vegetal promedio (\%) del período evaluado (2012-2015) de cada fracción. Prueba de Mann-Whitney $(n=419$, a $<0,05) *$ con diferencias significativas.

La presencia de estas malezas (B. punctulata, E. horridum y ONF) compiten con las especies forrajeras generando baja productividad de estos ambientes (Sione, et al., 2006; Sabattini, R.A., Ledesma, Sione, Fontana y Sabattini, J., 2014).

En diversas regiones de Argentina se ha evaluado el efecto nocivo del desarrollo de comunidades de especies arbustivas en el pastizal natural. Estudios indican que en la región Chaqueña Occidental y Monte Septentrional (Oyarzabal et al., 2018), la extracción forestal realizada entre fines del siglo XIX y mediados del siglo $X X$ ha desencadenado un proceso de sucesión secundaria resultando en la aparición de un bosque natural degradado, con un alto grado de invasión de arbustivas y un estrato de gramíneas muy pobre. Para los pastizales de la región del Noroeste Argentino, Martín (2014) considera problemático una cobertura de especies arbustivas que supere el $30 \%$, por las dificultades que representan para el manejo del rodeo y como competencia para el mantenimiento de las herbáceas forrajeras.

Los resultados globales son similares a los obtenidos en un bosque nativo del centro de Entre Ríos, donde la cobertura inicial de $B$. punctulata fue del $28 \%$, disminuyéndose al 5,8\% luego de 210 DDA por medio de una aplicación química terrestre con el mismo principio activo (Cottani y Sabattini, 2006). Trabajos de la región confirman la eficiencia de control sobre la arbustiva utilizando Picloram con el agregado de 2,4D en aplicaciones aéreas sobre bosques del espinal mesopotámico (Grancelli, Ludi, Casermeiro y Spahn, 1996; Marchesini, 2003).

Si bien al finalizar la evaluación se observan algunos ejemplares nuevos de $B$. punctulata, los mismos fueron originados por regeneración del banco de semilla. En el caso de las malezas perennes, presentan órganos subterráneos de reserva muy desarrollados, haciendo más difícil su control 
(Horowitz, 1972; Ghersa y Satorre, 1981; Hurle, 1998).

Teniendo en cuenta la dinámica anual, las forrajeras aumentaron su cobertura significativamente en las áreas tratadas respecto al testigo durante todos los años evaluados (Figura 4), oscilando con mínimos de $40 \%$ y máximos de $80 \%$ de cobertura vegetal. La fracción testigo se mantuvo con una tendencia constante durante la evaluación pero con oscilaciones interanuales muy marcadas. Este comportamiento pudo deberse por la combinación de dos causas: la modificación interanual de las precipitaciones y los cambios en el régimen de luz sobre el estrato herbáceo. Por un lado, es importante mencionar que la región presentó una variabilidad climática interanual y estacional muy marcada (Figura 2).

Durante los meses primaverales del 2012 las precipitaciones fueron muy por encima de lo normal $(102 \%)$, mientras que en los meses invernales del 2013 se presentaron por debajo de lo normal (69\%). En cambio, 2012 y 2015 tuvieron condiciones tendientes a la normalidad (Figura 2). Estas variaciones provocaron modificaciones sobre la composición florística del estrato herbáceo dominado por especies graminiformes de tipo C3 (Sabattini et al., 2002), que son altamente susceptibles a condiciones extremas de inundación o sequía. Por otro lado, la reducción de la cobertura de $B$. punctulata, especie cuya altura puede alcanzar los $2,5 \mathrm{~m}$, provoca un incremento sobre la entrada de luz, representando un ambiente propicio para el desarrollo de especies herbáceas como también de ONF (Burkart, 1969). Este comportamiento ha sido observado por Sabattini (com. pers.) donde el control de la arbustiva favoreció el desarrollo del M. macra Nees.

La fracción $B$. punctulata disminuyó desde la aplicación a $0 \%$ de cobertura luego de 40 DDA, con leves aumentos a partir de Julio de 2013 por una presión competitiva del estrato herbáceo coincidente con la sequía de ese momento. Durante todos los años del ensayo se obtuvo un control aceptable a muy bueno, medido indirectamente por la disminución significativa de su cobertura vegetal (Figura 4). Estos resultados permitirían mencionar que bajo estas condiciones ambientales (precipitaciones, manejo de la carga animal, utilización del principio activo y cobertura del estrato arbóreo), el control químico fue eficaz. Estudios realizados en otras regiones del mundo indican que la aplicación aérea en arbustivas permite disminuir sólo un $20 \%$ al primer año, y luego se requiere una intervención durante al menos siete años para detener el avance de las arbustivas por regeneración del banco de semillas (Miller \& Siriworakul, 1992; Cook, Setterfield \& Maddison, 1996). Los resultados con numerosos herbicidas han sido extremadamente variables $y$ dependen en gran medida de los factores ambientales, principalmente edáficos y climáticos que inciden en la efectividad de los productos químicos, afectando esencialmente su penetración y transporte. La traslocación de los herbicidas reguladores del crecimiento está estrechamente asociado con el transporte de fotosintatos; por lo tanto, uno o varios de los factores ambientales que influyen en dicho traslado tienen probablemente incidencia sobre la acción de los herbicidas sistémicos (Fernández, Leguizamón y Acciaresi, 2015).

Si bien B. punctulata es nativa del Espinal Argentino (Oyarzabal et al., 2018), han sido referidas como invasoras en bosques nativos y pastizales naturales degradados de la Mesopotamia (Casermeiro y Spahn, 1999; Marchesini, 2003) y en otras regiones argentinas con algún tipo de deterioro como erosión hídrica (Gaspari y Bruno, 2003). Pertenece a la familia Asterácea, de ciclo primavero-estival, cuyo vigoroso sistema radical actúa como un importante órgano de reserva que le permite resistir y subsistir a las más variadas condiciones adversas, tales como los cortes por desmalezado mecánico. Esto, sumado a su abundante floración y producción de semillas conlleva a que esta especie alcance notables niveles de invasión y la consiguiente ocupación gradual del terreno (Marchesini, 2003).

Respecto E. horridum, durante el primer año se observó un descenso significativo de la cobertura en las áreas con tratamiento químico, pero a partir del segundo año no sé registraron diferencias significativas (Figura 4). Sin embargo, la cobertura de esta especie aumentó en las parcelas tratadas respecto al testigo, debido al nuevo nicho ecológico generado por la disminución de la cobertura de $B$. punctulata y además, a causa de la falta de competencia por el resto de las fracciones, como por ejemplo las forrajeras.

El aumento en la cobertura de las ONF a partir del segundo año respecto al primero, tanto en parcelas testigo como en tratadas, se debió a un sobrepastoreo realizado por cuestiones logísticas y 
operativas del ANP-El Carayá. La intensidad y presión de pastoreo pudo ocasionar la disminución no significativa de la cobertura vegetal del $E$. horridum en ambos tratamientos a partir del tercer y cuarto año de evaluación (Figura 4).
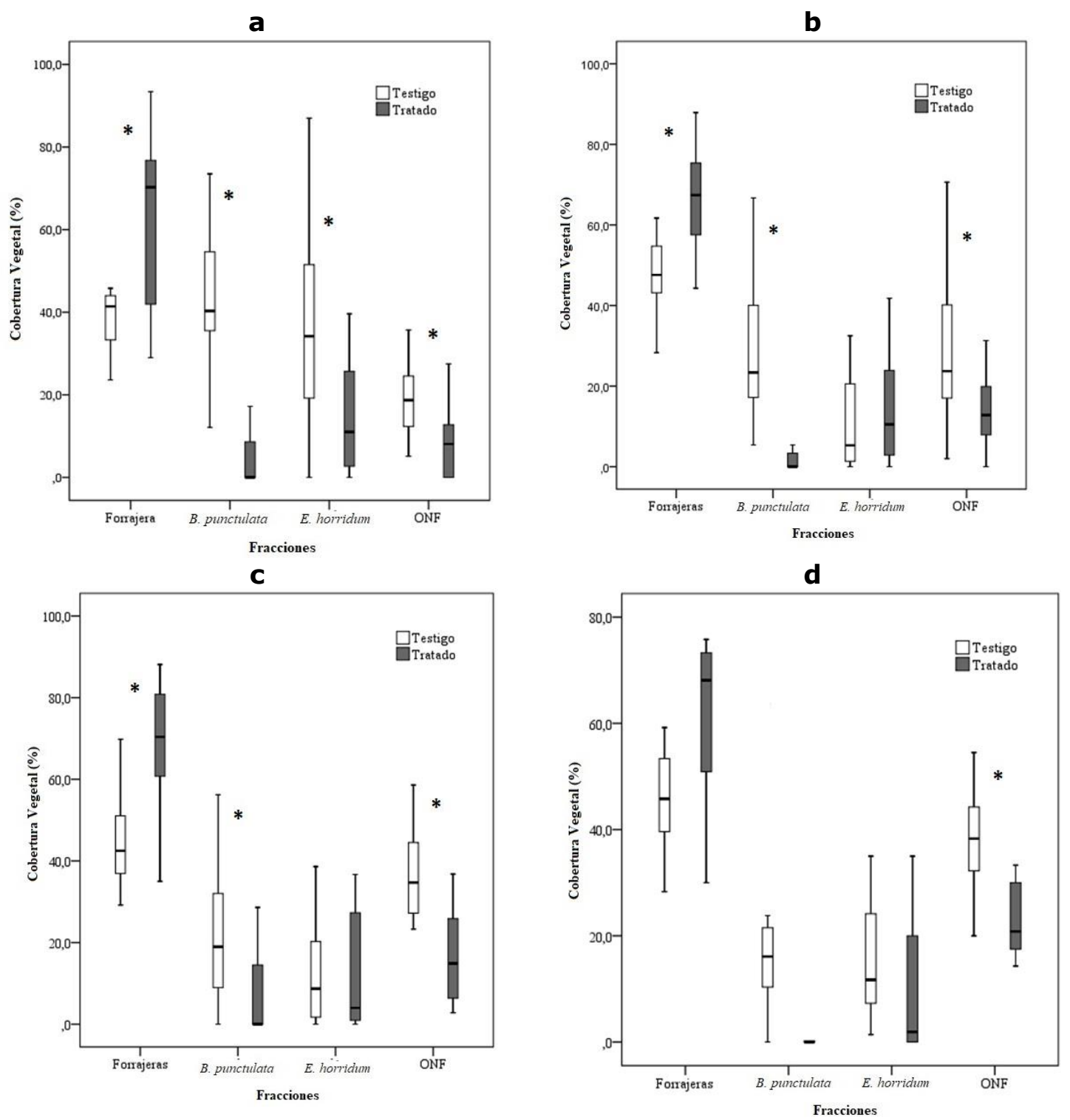

Figura 4. Cobertura vegetal promedio (\%) anual de las fracciones evaluadas en el sector testigo ( $\square$ ) y tratado químicamente ( $\mathbf{a})$. Prueba de Mann-Whitney $(n=419$, a $<0,05) *$ con diferencias significativas. a-Año 2012: Forrajeras $Z=-2,401, p=0,016 ;$ B. punctulata $Z=-3,871, p<0,05$; E. horridum $Z=-2,075, p<0,05$; y ONF $Z=-2,174, p<0,05$. b-Año 2013: Forrajeras $Z=-3,692$, $\mathrm{p}<0,05$; $B$. punctulata $Z=-4,499, p<0,05 ;$ E. horridum $Z=-0,707, p=0,480$; y ONF $Z=-2.053$, $\mathrm{p}<0.05$. c-Año 2014: Forrajeras $Z=-3,366, \mathrm{p}=0,001 ; B$. punctulata $Z=-2.266, \mathrm{p}<0,05 ; E$. horridum $Z=-0.303, p=0,762$; y ONF $Z=-3,529, p<0,001$. d-Año 2015: Forrajeras $Z=-2,388$, $\mathrm{p}=0,017$; B. punctulata $\mathrm{Z}=-1,944, \mathrm{p}=0,055$; $E$. horridum $\mathrm{Z}=-1.851, \mathrm{p}=0,064$; y ONF $Z=-$ 2.505, $\mathrm{p}<0,05$.

En el área tratada químicamente se observó que la cobertura se mantuvo constante durante el período analizado, lo cual es posible considerarlo como indicador de que el sistema presentó una estabilidad en el tiempo. Esta especie es una maleza agresiva y su presencia en los pastizales reduce la receptividad al dificultar el acceso del ganado, porque sus hojas están provistas de espinas, y además se reproduce por semillas y por rizomas (Elizalde, Lallana, M.C. y Lallana, V.H., 1997; 
Lallana, V.H., Elizalde, Lallana, M.C., Billard y Cristaldo, 2003). Otros trabajos indican un resultado similar a este trabajo, no teniendo efectos importantes en el control de la parte aérea (Lallana et al., 1998; 2002).

La persistencia en el control de las arbustivas observada durante el período evaluado, pone en ventaja a la práctica empleada frente a otras estrategias de control, por ejemplo, a través de la eliminación mecánica de los arbustos. Marchesini (2003) indica que la eliminación mecánica resulta una práctica de efecto inmediato pero que debe realizarse al menos con una frecuencia anual, dado que por lo general las malezas rebrotan rápidamente haciendo uso de sus reservas. En este sentido se ha demostrado en el Chaco Semiárido Argentino que, transcurridos 10 años de la práctica mecánica, los arbustos recuperan su posición en la estructura del bosque (Brassiolo, Lorea, González y Zárate, 2008).

\section{Composición florística}

Durante todo el periodo considerado no se observaron diferencias significativas en las áreas tratadas químicamente al finalizar las evaluaciones en las especies herbáceas de mayor abundanciacobertura del pastizal natural estudiado respecto al testigo (Tabla 1).

Tabla 1. Cobertura promedio (\%) anual de las especies herbáceas con mayor frecuencia del pastizal natural en el testigo $(T)$ y tratado químicamente $(Q)$. Test de Kruskal Wallis $(a>0,05) *$ medias con diferencias significativas.

\begin{tabular}{|c|c|c|c|c|c|c|c|}
\hline Especie herbácea & & 2012 & 2013 & 2014 & 2015 & Media & $\mathbf{w} / p$ \\
\hline Ciperáceas & $\begin{array}{l}\text { T } \\
\mathrm{Q}\end{array}$ & $\begin{array}{c}0,50 \\
15,01\end{array}$ & $\begin{array}{l}1,89 \\
4,57\end{array}$ & $\begin{array}{l}0,00 \\
0,59\end{array}$ & $\begin{array}{l}2,13 \\
3,22\end{array}$ & $\begin{array}{l}1,69 \\
5,33\end{array}$ & $0,46 / 0,4988$ \\
\hline Desmodium spp. & $\mathrm{T}$ & $\begin{array}{l}7,94 \\
0,58\end{array}$ & $\begin{array}{c}11,11 \\
0,56\end{array}$ & $\begin{array}{l}0,75 \\
0,75\end{array}$ & $\begin{array}{l}2,61 \\
3,51\end{array}$ & $\begin{array}{l}6,20 \\
4,20\end{array}$ & $0,07 / 0,7916$ \\
\hline Paspalum spp. & $\begin{array}{l}\mathrm{T} \\
\mathrm{Q}\end{array}$ & $\begin{array}{l}9,05 \\
6,74\end{array}$ & $\begin{array}{c}22,80 \\
23,8\end{array}$ & $\begin{array}{c}19,17 \\
9,83\end{array}$ & $\begin{array}{c}13,40 \\
7,92\end{array}$ & $\begin{array}{l}20,99 \\
17,77\end{array}$ & $0,46 / 0,4969$ \\
\hline Piptochaetium spp. & T & $\begin{array}{l}8,62 \\
2,23\end{array}$ & $\begin{array}{c}7,47 \\
21,11\end{array}$ & $\begin{array}{l}8,29 \\
3,50\end{array}$ & $\begin{array}{c}13,12 \\
4,78\end{array}$ & $\begin{array}{l}9,02 \\
8,54\end{array}$ & $0,06 / 0,8054$ \\
\hline Setaria spp. & T & $\begin{array}{l}14,44 \\
17,22\end{array}$ & $\begin{array}{c}5,93 \\
15,91\end{array}$ & $\begin{array}{c}21,42 \\
5,21\end{array}$ & $\begin{array}{l}4,18 \\
3,10\end{array}$ & $\begin{array}{l}11,69 \\
10,80\end{array}$ & $0,04 / 0,8355$ \\
\hline Nassella spp. & $\begin{array}{l}\mathrm{T} \\
\mathrm{Q}\end{array}$ & $\begin{array}{l}37,29 \\
40,89\end{array}$ & $\begin{array}{l}16,40 \\
13,51\end{array}$ & $\begin{array}{l}2,29 \\
6,29\end{array}$ & $\begin{array}{c}11,55 \\
8,28\end{array}$ & $\begin{array}{l}18,69 \\
18,71\end{array}$ & $0,06 / 0,8067$ \\
\hline
\end{tabular}

Estos resultados permiten afirmar el nivel de selectividad del principio activo aplicado sobre las especies gramíneas del pastizal natural en bosques nativos del Espinal Mesopotámico que son de alto valor forrajero. Estudios realizados para el control de Baccharis spp. con Picloram + 2,4-D indican buena efectividad inclusive a dos años de realizada la pulverización, sin provocar efectos fitotóxicos de importancia en el pastizal natural (Sabattini et al., 2011). Si bien no se observaron diferencias significativas respecto a la composición florística entre el área de control químico y el testigo, queda el interrogante acerca de la nueva organización de la comunidad del pastizal a largo plazo.

Las especies invernales (Nassella spp. y Piptochaetium spp.) no mostraron diferencias entre testigo y el tratamiento químico durante la evaluación, sin embargo, la cobertura presentó una alta variabilidad interanual. En cambio, las gramíneas de ciclo estivo-primaveral (Paspalum spp. y Setaria spp.) presentaron leve descenso no significativo (Tabla 1 ) en el tratado respecto al testigo desde mediados del año 2013. Este comportamiento puede deberse a una mayor preferencia en el forrajeo del ganado animal y escasa recuperación del pastizal posterior a una defoliación.

En este sentido, si bien el pastoreo fue de baja intensidad, diferentes estudios han demostrado que es una de las causas en la modificación de la composición florística en los pastizales naturales (Morici et al., 2003; Bertiller, Bisigato, Carrera y Del Valle, 2004; Bisigato, Bertiller, Ares, Pazos \& Pugnaire, 2005), disminuyendo la cobertura (Bisigato \& Bertiller, 1997), el vigor, la supervivencia y producción de semillas (O'Connor, 1991; Privitello, Gabutti, Harrison, Sager y Romero, 2000).

Contrario a la afirmación anterior, en pastizales con larga historia de pastoreo, la diversidad es máxima en presencia de los herbívoros y en general, no se 
observa la invasión de especies exóticas ni la pérdida de especies nativas aún a una alta carga ganadera (Sabattini et al., 2008).

Por otro lado, la presencia de Paspalum spp. y Nassella spp., son indicadoras de buena calidad de sitios, especies de la familia Poaceae, aseguran la posibilidad de recobrar una buena condición del pastizal natural cercana a la vegetación original en equilibrio con las condiciones climáticas, edáficas y de herbivoría de especies nativas (Landi y Galli, 1984).

Respecto a las leguminosas, representada por Desmodium spp., no se observó una disminución significativa en el porcentaje de cobertura en el tratamiento $(4,2 \%)$ respecto al testigo $(6,2 \%)$. La disminución registrada se la atribuye a una mayor accesibilidad y elevada preferencia por su valor proteico por parte del ganado bovino.

Por otro lado, se registró un aumento en la cobertura de las ciperáceas durante todo el ensayo en el sector tratado químicamente pero no fue significativo (Tabla 1), destacándose el año 2012, atribuible posiblemente a las excesivas precipitaciones primaverales del período (Figura 2). Estas especies se encuentran adaptadas a condiciones de humedad elevadas, y en este caso en particular, aumentaron su cobertura porque se mejoraron las condiciones del nicho ecológico como, por ejemplo, mejor régimen de luz y, posiblemente, mayor contenido de nutrientes en el suelo (Sione et al., 2006).

\section{Biomasa vegetal}

El control de la fracción de $B$. punctulata comprobado por su disminución significativa de la cobertura vegetal $(Z=-6,453 ; p<0,001)$, permitió aumentar la productividad primaria promedio del pastizal natural en un $77 \%$ durante el período de evaluación en las áreas tratadas $(4.302 \pm 2.586 \mathrm{~kg}$ MS ha-1 año-1) respecto del testigo (2.429 \pm 1.630 kg MS ha-1 año-1).

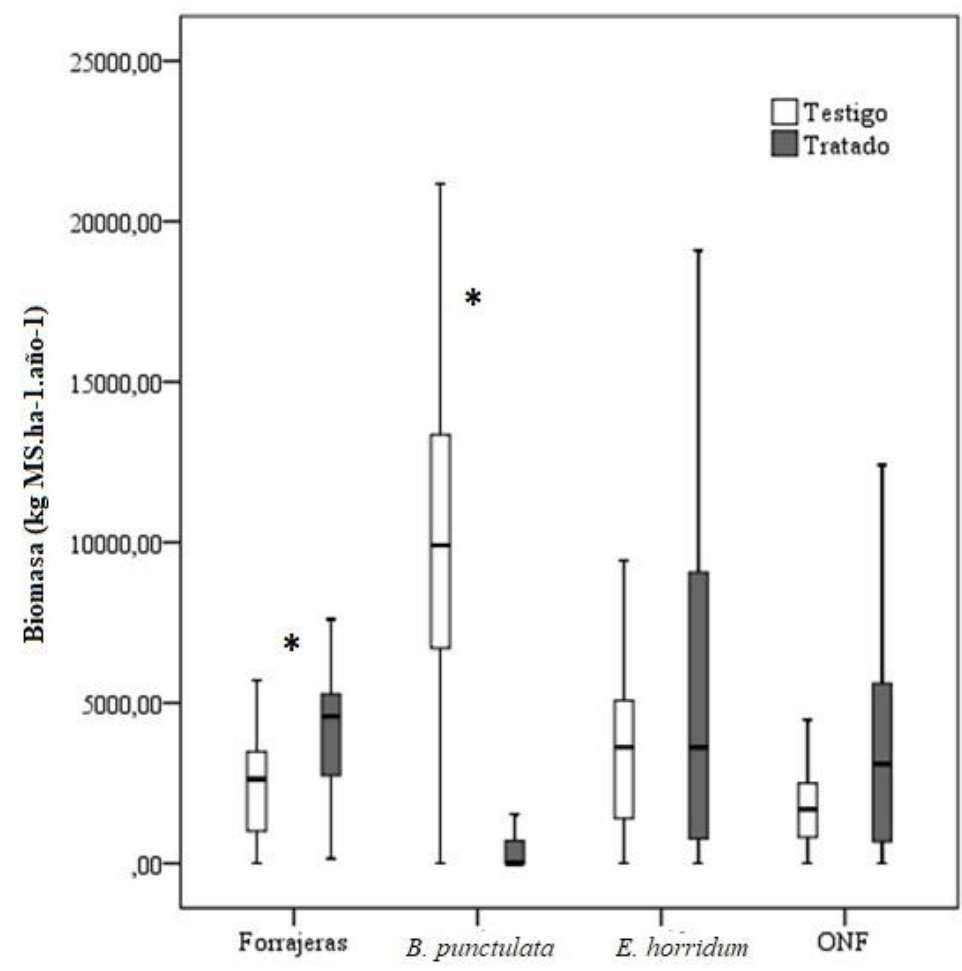

Fracciones

Figura 5. Biomasa vegetal promedio (\%) anual del período evaluado (2012-2015) de cada fracción (testigo ( $\square$ ) y tratado químicamente ( $\square)$. Prueba de Mann-Whitney $(n=419$, a $<0,05) *$ con diferencias significativas. Nota: la productividad primaria del Año 2012 se consideró tomando el período Mayo a Diciembre, y en el Año 2015 el período de Enero a Junio. 
La productividad interanual registró el mismo comportamiento. Similar comportamiento se observó en las fracciones de Forrajeras y ONF. Esto denota la capacidad de recuperación que tienen las especies herbáceas del pastizal natural.

Bovey, Haas \& Meyer (1972) hallaron una excelente recuperación en la producción de forrajes con posterioridad al control de varias arbustivas indeseables en Texas. Los resultados globales presentan similitudes con aplicaciones químicas sobre bosques nativos de la región (Sosa, 2012; Arbello \& Mhor, 2013; Kruger, 2014), como también sobre regiones áridas y semiáridas donde la competencia entre el estrato arbustivo y el herbáceo es aún más crítica.

Por otro lado, las nuevas condiciones permitirían adicionar potencialmente una productividad secundaria de $68 \mathrm{~kg}$ carne ha-1 año-1, considerando los requerimientos para criar un ternero hasta el destete a los 6 meses (Carillo, 1997). En este sentido, la utilización de esta técnica de control, permitiría aumentar la producción ganadera de la región tal como plantean investigaciones en otras regiones del mundo (Tiedemann \& Klemmedson, 1977; Jacoby \& Meadors, 1982; Ueckert, Jacoby \& Hartmann, 1982).

La productividad anual promedio registrada de $B$. punctulata en condiciones naturales en un bosque nativo fue de $10.484 \pm 7.946 \mathrm{~kg} \mathrm{MS} \mathrm{ha-1} \mathrm{año-1,}$ siendo significativamente inferior cuando se realiza una aplicación química aérea. Sin embargo, las fracciones $E$. horridum y ONF no presentaron diferencias significativas (Figura 5). El aumento significativo de ambas fracciones en el año 2014 se debió, por un lado, a la mayor iluminación de los estratos medios e inferiores como consecuencia de la eliminación de las arbustivas y, por otro lado, a la falta de competencia de especies forrajeras por sobrepastoreo durante el período de mayor crecimiento del E. horridum y otras especies como Eupatorium spp., y Pterocaulon spp. Elliott.

\section{CONCLUSIONES}

Los resultados de este trabajo comprueban que la utilización de una técnica de control químico aéreo permitiría mejorar el pastizal natural de un bosque nativo del Espinal argentino con arbustivas. Esta recuperación se refleja por un aumento significativo de la cobertura y biomasa en las áreas tratadas de las especies forrajeras respecto al testigo y una disminución significativa de la fracción B. punctulata y ONF.

Por otro lado, la composición florística de las especies herbáceas más conspicuas del pastizal natural no presentó diferencias significativas en el sector tratado respecto al testigo, comprobando indirectamente la selectividad del principio activo sobre la mayoría de las gramíneas del tapiz. De esta manera la técnica puede encuadrarse dentro de los parámetros de sustentabilidad ecológico-ambiental considerando solo aspectos de la vegetación.

La técnica permitió incrementar además, la superficie útil de pastoreo, mejorando la productividad secundaria de la empresa ganadera, y disminuyendo la concentración de principio activo por el tiempo de persistencia sobre el control de arbustivas.

\section{AGRADECIMIENTOS}

Este trabajo fue realizado en el marco del Convenio de Asistencia Técnica entre la FCA-UNER y Las Taperitas SA "Sustentabilidad productiva y ambiental Establecimiento "El Carayá" (Feliciano, Entre Ríos): aplicación de prácticas de control y manejo para la recuperación de áreas de pastoreo en montes nativos".

Los autores agradecen al personal del ANP-El Carayá por el apoyo en las tareas de campo, y logística al momento de la aplicación, como también a los Sres. Lucas Sandobal, Javier Williner y Gastón Funes las instalaciones del campo. Además, a los Ings. Agrs. Alejandro Kruger, Mauro Lindt, y Raul Facendini por colaborar en las evaluaciones y procesamiento de muestras.

\section{REFERENCIAS BIBLIOGRÁFICAS}

Anadón, J.D., Sala, O.E., Turner, B.L. \& Bennett, E.M. (2014). Effect of woody-plant encroachment on livestock production in North and South America. Proceedings of the National Academy of Sciences of the United States of America, 111(35), 1294812953.

Archer, S. (1990). Development and Stability of Grass/Woody Mosaics in a Subtropical Savanna Parkland, Texas, U. S. A. Journal of Biogeography, $17(4 / 5), 453-462$

Bailey, R.G. \& Ropes, L. (1998). Ecoregions: The Ecosystem Geography of the Oceans and 
Continents: with 106 illustrations, with 55 in color. New York : Springer, pp 180.

Beever, E.A., Wilkening, J.L., McIvor, D.E., Weber, S.S. \& Brussard, P.F. (2008): American pikas (Ochotona princeps) in northwestern Nevada: a newly discovered population at a lowelevation site. Western North American Naturalist, 68(1), 8-14.

Bertiller, M.B., Bisigato A.J., Carrera, A.L. y Del Valle, H.F. (2004). Estructura de la vegetación y funcionamiento de los ecosistemas del Monte Chubutense. Boletín de la Sociedad Argentina de Botánica, 39(3-4):139-158.

Bisigato, AJ \& Bertiller, MB. (1997). Grazing effects on patchy dryland vegetation in northern Patagonia. Journal of Arid Environmental, 36: 639-653.

Bisigato, A.J., Bertiller, M.B., Ares, J.O., Pazos, G.E. \& Pugnaire, F. (2005). Effect of grazing on plant patterns in arid ecosystems of Patagonian Monte. Ecography, 28(5): 561-572

Böker, R., Gulielmetti, B. y Knudtsen, O. (1989). Control de malezas leñosas en pasturas. Revista de la Sociedad Rural de Jesús María, 53:45-48.

Bovey, R.W., Haas, R.H. \& Meyer, R.E. (1972). Daily and seasonal response of huisache and Macartney rose to herbicides. Weed Science, 20(6), 577-580.

Brassiolo, M., Lorea, L., González, D.P. y Zárate, M.H. (2008). Reacción del estrato arbustivo a diferentes intervenciones y presencia de ganado vacuno, en el Chaco Semiárido. Quebracho Revista de Ciencias Forestales, (No 16), 51-61.

Braun-Blanquet, J. (1979). Fitosociología: bases para el estudio de las comunidades vegetales. Madrid, España: Blume, 820 p.

Burkart, A. (1969). Leguminosas nuevas o críticas, VII. Darwiniana 15 (3-4), 501-549.

Casermeiro, J. y Spahn, E. (1999). Sistemas agroforestales para pequeños productores de zonas húmedas. Santa Fe, Argentina, Secretaría de Industria de la Nación. 214 p.

Casermeiro, J., Spahn, E., De Petre, A. (2001). La integración extensión-docencia-investigación como estrategia metodológica para la educación y el desarrollo sostenible de los recursos naturales. En: 1er Congreso y 5ta Jornada Regional sobre Manejo de Pastizales Naturales 9-11 de agosto. San Cristóbal, Santa Fe, Argentina, 108-109 p.

Cook, G.D., Setterfield, S.A \& Maddison, J.P. (1996). Shrub invasion of a tropical wetland:
Implications for weed management.Ecological Applications, 6(2), 531-537.

Cottani, F. y Sabattini, R.A. (2006). Manejo y control de arbustivas en un pastizal con alta carga animal en pastoreo rotativo. Revista Científica Agropecuaria, 10(2), 109-120

Cozzo, D. (1979). Arboles forestales, maderas y silvicultura de la Argentina. Enciclopedia Argentina de Agricultura y Jardinería. Buenos Aires, Argentina: Acme, $156 \mathrm{p}$.

Donlan, C.J., Tershy, B.R. \& Croll, D.A. (2002). Islands and introduced herbivores: conservation action as ecosystem experimentation. Journal of Applied Ecology, 39, 235-246

Elizalde, J.H.I., Lallana, M.C. y Lallana V.H. (1997). Reproducción sexual y asexual de Eryngium paniculatum - Apiaceae -"caraguatá". En: Actas XIII Congreso Latinoamericano de Malezas. Buenos Aires, 161-170.

Enloe, S. \& Loewenstein, N. (2013) Control Options For Chinese Privet. rep. ANR-1468. Alabama, Estados Unidos: Cooperative Extension, 254 p.

FAO. (2007). Situación de los Bosques del Mundo 2007. Roma, Italia: FAO, 143 p.

FAO. (2009). Situación de los Bosques del Mundo 2009. Roma, Italia: FAO, 158 p.

Fernández, O.A., Leguizamón, E.S. y Acciaresi, H.A. (2015). Malezas e Invasoras de la Argentina. Tomo I: Ecología y Control. Bahía Blanca, AR: UNS-REUN, $945 \mathrm{p}$.

Fumagalli, A.E. y Kunst, C.G.R. (2002). Producción de carne vacuna en el NOA. ¿Cómo mejorar la oferta forrajera de los sistemas de cría? IDIA XXI Revista de Información sobre Investigación y Desarrollo Agropecuario, 2(2):73-78.

Gann, B., Thompson, L. \& Schuler, J.L. (2012). Control and management of eastern baccharis in a recently established bottomland hardwood plantation. In: Proceedings of the 16th Biennial Southern Silvicultural Research Conference. e-Gen. Tech. Rep. SRS-156. Asheville, NC: US Department of Agriculture Forest Service, Southern Research Station, $122-126 \mathrm{p}$.

Gaspari, F.J. y Bruno, J.E. (2003). Diagnóstico de degradación ambiental por erosión hídrica en la cuenca del arroyo Napostá Grande. Ecología Austral, 13(1), 109-120. 
Getsinger, K.D., Madsen, J.D., Koschnick, T.J. \& Netherland, M.D. (2002). Whole lake fluridone treatments for selective control of Eurasian waterm ilfoil: I. Applicationstrategy and herbicide residues. The Lake and Reservoir Management Journal, 18 (3), 181-190

Ghersa, C.M. y Satorre, E H. (1981). La dinámica de la población de rizomas de sorgo de alepo en relación con los sistemas de control más frecuentes. Revista Facultad de Agronomía 2(3), 133-138.

Grancelli, R., Ludi, R., Casermeiro, J. y Spahn E. (1996). Control químico aéreo de chilca (Baccharis spp.) en un sistema ganadero de María Grande. EEA INTA Paraná. En: Jornada Técnica de Producción Ganadera - Resultados de experiencias en campo de productores en el área de la EEA. Paraná. (19941996). 23 de mayo. (Serie Extensión, No 10). Paraná : INTA, p. 43-49.

Graz, F.P. (2008) The woody weed encroachment puzzle: gathering pieces. Ecohydrology, 1, 340348.

Grime J.P. (1977). Evidence for the Existence of Three Primary Strategies in Plants and Its Relevance to Ecological and Evolutionary Theory. The American Naturalist, 111(982):1169-1194.

Hansen, M.C., Potapov, P.V., Moore, R., Hancher, M., Turubanova, S.A., Tyukavina, A., ...Townshend, J.R.G (2013). High-Resolution Global Maps of 21stCentury Forest Cover Change. Science 342, 850853

Helm, A. Oja, T., Saar, L., Takkis K., Talve, T. \& Pärtel, M. (2009). Human influence lowers plant genetic diversity in communities with extinction debt. Journal of Ecology, 97, 1329-1336.

Hobbs, R.J. \& Huenneke, I.F. (1992). Dusturbance, diversity, and invasión: implications for conservation. Conservation Biology, 6: 324-337.

Holmes, D.M. (1998). Management and ecology of Willow Baccharis in the Texas rolling plains. Thesis to Master of Science. Texas : Faculty of Texas Teach University, 148 pp.

Holmgren, M. (2002). Exotic herbivores as drivers of plant invasion and switch to ecosystem alternative states. Biological Invasions, 4, 25-33.

Horowitz, M. (1972). Development of Cynodon Dactylon (L.). Pers. Weed Research, 12(3), 207220.
Hurle, K. (1998). Present and future developments in weed control - A view from weed science. Planzenschutz-Nachrichten Bayer, 51, 109-137.

INTA. (1980). Suelos y erosión de la Provincia de Entre Ríos: plan mapa de suelos. (Serie Relevamiento de Recursos Naturales N01). Tomo 1. Paraná, Argentina, 109 p.

INTA. (1986). Carta de suelos de la República Argentina. Departamento Feliciano, Provincia de Entre Ríos: plan mapa de suelos de la Provincia entre Ríos. Memoria técnica, con mapa de suelos y de vegetación natural a esc. aprox. 1:100.000. E.E.A Paraná. (Serie Relevamiento de Recursos Naturales, No 3), 96 pp.

Jacoby, P.W. \& Meadors, C.H. (1982). Control os Sand Shinnery Oak (Quercus havardii) with Pelleted Picloram and Tebuthiuron. Weed Science, 30, 594597.

Kearns, C.A., Inouye, D.W. \& Waser, N.M. (1998). Endangered mutualisms: the conservation of plantpollinator interactions. Annual review of ecology and systematics,29(1), 83-112.

Kruger, R.A. (2014). Manejo y control de Baccharis punctulata (chilca blanca) para la recuperación productiva del pastizal natural en un monte sometido a pastoreo rotativo con alta carga instantánea. Trabajo Final de Graduación Ingeniería Agronómica. Oro Verde, Argentina: Facultad de Ciencias Agropecuarias UNER, 78 pp.

Lallana, V., Faya De Falcón, L., Elizalde, J., Lallana, M.C., Sabattini, R.A., Billard, C., ... Anglada, M. (1998). Control integrado del "caraguatá" en un campo de San Gustavo (La Paz - Entre Ríos). (Serie de Extensión. Informe técnico, n 16). Paraná, Argentina: INTA, $54 \mathrm{p}$.

Lallana, V.H., Elizalde, J.H.I., Lallana, M.C., Billard, C. y Cristaldo, M. (2003). Biología y control de de rizomas de Eryngium paniculatum ("caraguatá"). 2do. Informe de avance. PID 2076. Facultad de Ciencias Agropecuarias. UNER, 51 p.

Lallana, V.H., Lallana, M.C., Elizalde, J.H.I., Billard, C.E., Rochi, G. y Cristaldo, M. (2002). Control de "caraguatá" (Eryngium paniculatum) en pastizales naturalizados de Entre Ríos. Ciencia, Docencia y Tecnología, 25, 211-234.

Landi, M.P. y Galli, I. (1984). Introducción al manejo del campo natural en la provincia de Entre Ríos. Concepción del Uruguay, Argentina (Serie Producción Vegetal, Boletín Técnico, No 24): INTA., $20 \mathrm{p}$. 
Marchesini, E. (2003). Control de chilca. Concepción del Uruguay, Argentina. (Serie técnica $n^{\circ}, 87$ ) : INTA, 25 p.

Martín, G.O. (2014). Técnicas de refinamiento y recuperación de pastizales (Serie Didáctica, N85).. San Miguel de Tucumán, Argentina: Facultad de Agronomía y Zootecnia, Universidad Nacional de Tucumán, $64 \mathrm{p}$

Matteucci, M y Colma, P. (1982). Metodología para el estudio de la vegetación (Serie Biología). Washington DC, Estados Unidos: OEA, 168 p.

Miller, I.L. \& Siriworakul, M. (1992). Herbicide research and recommendations for control of Mimosa pigra. En: Harley, K.L.S. A Guide to the Management of Mimosa pigra. Canberra, Australia: CSIRO, $89 \mathrm{p}$.

Moleele, N.M., Ringrose, S., Matheson, W. \& Vanderpost, C. (2002). More woody plants? The status of bush encroachment in Botswana's grazing areas. J. Environ. Manage., 64 (1), 3-11.

Morici, E.F.A., Ernst, R., Kin, A., Estelrich, D., Mazzola, M. y Poey, S. (2003). Efecto del pastoreo en un pastizal semiárido de Argentina según la distancia a la aguada. Archivos de Zootecnia, 52(197), 59-66.

O'Connor, T.G. (1991). Influence of rainfall and grazing on the compositional change of the herbaceous layer of a sandveld savanna. Journal of the Grassland Society of Southern Africa, 8(3), 103109.

Oyarzabal, M., Clavijo, J., Oakley, L., Biganzoli, F., Tognetti, P., Barberis, I., ...León, R.J.C. (2018). Unidades de vegetación de la Argentina. Ecología Austral, 28(1), 40-63.

Parker, M.A., Malek, W. \& Parker, I.M. (2006). Growth of an invasive legume is symbiont limited in newly occupied habitats. Diversity and Distributions, 12, 563-571.

Pettit, N.E., Froend, R.H. \& Ladd, P.G. (1995). Grazing in remnant woodland vegetation: changes in species composition and life form groups. Journal of vegetation science, 6, 121-130

Privitello, M.J.L., Gabutti, E.G., Harrison, R.U., Sager, R.L. y Romero, M.B. (2000). Efecto de dos intensidades y cuatro frecuencias de corte sobre la productividad, vigor y persistencia de Piptochaetium napostaense (Speg.) Haeckel. Revista Argentina de Producción Animal, 20: 23-127.
Randall, J. (2013). Chemical Control of Unwanted Shrub \& Tree Vegetation. Ames, Iowa, Estados Unidos, IOWA State University. 7p.

Renz, M. (2013). Herbicide effectiveness on invasive plantas in Wisconsin. Wisconsin. Estados Unidos: University of Wisconsin - Cooperative Extension, $4 p$.

Reynolds, J.F., Maestre, F.T., Kemp, P., StaffordSmith, M. \& Lambin, E. (2007). Natural and human dimensions of land degradation in drylands: causes and consequences. Berlín, Alemania: Springer, 268 p.

Rojas, A. y Saluso, J. (1987) Informe climático de la provincia de Entre Ríos. EEA-INTA Paraná. Publicación Técnica No 14, 20 p.

Sabattini, J.A., Sabattini, R.A. y Ledesma, S. (2015). Caracterización del bosque nativo del centro norte de Entre Ríos (Argentina). Agrociencia. 19, 816.

Sabattini, R.A., Wilson M.G., Muzzachiodi N. y Dorsch, A.F. (1999). Guía para la caracterización de agroecosistemas del centro-norte de Entre Ríos. Revista Científica Agropecuaria, 3, 7-19

Sabattini, R.A., Muzzachiodi N. y Dorsch, A.F. (2002). Manual de Prácticas de Manejo del Monte Nativo. Oro Verde, Argentina: UNER, 56 p.

Sabattini, R.A., Sione, S., Ledesma, S., Muracciole, B., Cottani, F. y Fortini, C. (2008). Análisis de la diversidad florística y de los tipos productivos del pastizal natural en un monte nativo bajo pastoreo rotativo (Entre Ríos, Argentina). Revista Científica Agropecuaria., 12(1), 5-13.

Sabattini, R.A., Ledesma, S.G., Sione, S.M.J., Fontana, E. y Sabattini, J.A. (2011). Recuperación del pastizal natural degradado en un monte nativo sometido a desarbustado mecánico. Informe Final PID UNER 2129. Entre Ríos, Argentina: Facultad de Ciencias Agropecuarias, UNER, $51 \mathrm{p}$.

Sabattini, R.A., Ledesma, S.G., Sione, S.M., Fontana E. y Sabattini, J. (2014). Recuperación del pastizal natural degradado en un monte nativo sometido a desarbustado mecánico. Ciencia, Docencia y Tecnología UNER, 4 (4), 20-36.

Safriel, U. \& Adeel, Z. (2005). Dryland systems. Ecosystems and Human Well-Being, Current State and Trends. Washington, Estados Unidos: Island Press, $658 \mathrm{p}$. 
Scholes, R. \& Archer, S. (1997). Tree-grass interactions in savannas. Annual Review of Ecology, Evolution, and Systematics, 28, 517-544.

Sione, S.M., Sabattini, R.A., Ledesma, S.G., Dorsch, A.F. y Fortini, C. (2006). Caracterización florística y es-tructural del estrato arbustivo de un monte en pastoreo. Revista Científica Agropecuaria, 10 (1), 59-67

Sosa, F.S. (2012). Manejo y control del espartillo amargo (Melica macra Ness) para la recuperación productiva del pastizal natural en un monte sometido a pastoreo rotativo con alta carga instantánea. Trabajo Final de Graduación Ingeniería Agronómica. Oro Verde, Argentina: Facultad de Ciencias Agropecuarias, UNER, 84 pp.

Steinfeld H., Gerber P., Wassenaar T., Castel V., Rosales M. \& Haan C. de. (2006). Livestock's long shadow: environmental issues and options. Rome, Italy : FAO,. 390 pp.

Stohlgren, T.J., Schell, L.D. \& Vanden Heuvel, B. (1999). How grazing and soil quality affect native and exotic plant diversity in Rocky Mountain grasslands. Ecological applications, 9(1), 45-64.

Tiedemann, A.R. \& Klemmedson, J.O. (1977). Effect of Mesquite Tree on Vegetation and Soil in the Desert Grassland. Journal Range Management, $30(5), 361-367$

Tighe, M., Reid, N., Wilson, B. \& Briggs, S.V. (2009). Invasive native scrub and soil condition in semi-arid south-eastern Australia. Agric. Ecosyst. Environ., $132(3 / 4), 212-222$.

Tilman D., B.C. Hill, C. \& Lehman (2006). Carbonnegative biofuels from low-input high-diversity grassland biomass. Science., 314(5805), 15981600

Tolozano B., Pisani JM y Puricelli E.C. (2017). Control mecánico y químico de Tessaria dodoneifolia (Hook. et Arn.) Cabrera (chilca dulce). Revista de Investigaciones Agropecuarias., 43(2), 128-134.

Ueckert, D.N., Jacoby, P.W. \& Hartmann, S. (1982). Tarbush and forage response to selected pelleted herbicides in the western Edwards Plateau. Texas. Agr. Exp. Sta. Bull, 1393, 6p.

Villalobos, A.E de. (2013). El sobrepastoreo del Ganado doméstico como disparador de la arbustización. BioScriba, 6(1), 51-57

Vitousek, P.M. \& Walker, L.R. (1989). Biological invasion by Myrica faya in Hawai'i: plant demography, nitrogen fixation, ecosystem effects. Ecological monographs, 59(3), 247-265.

Wilcox, B.P. \& Thurow, T.L. (2006). Emerging issues in rangeland ecohydrology: vegetation change and the water cycle. Rangeland Ecology \& Management, 59(2), 220-224.

Zimdahl, R.L. (2004). Weed-crop competition: a review. Ames, Estados Unidos: Wiley-Blackwell Publishing, $220 \mathrm{p}$. 